\title{
RELATIONSHIP BETWEEN THE MEET AND JOIN OPERATORS IN THE LATTICE OF GROUP TOPOLOGIES
}

\author{
BRADD CLARK AND VICTOR SCHNEIDER
}

\begin{abstract}
Let $L(G)$ be the lattice of all topologies on the group $G$ which make $G$ into a topological group. If $\tau_{1}$ and $\tau_{2}$ are Hausdorff group topologies and $\tau_{1} \vee \tau_{2}$ is the discrete topology, then $\tau_{1} \wedge \tau_{2}$ is a Hausdorff topology. If $\tau_{1}$ and $\tau_{2}$ are locally compact Hausdorff group topologies, then $\tau_{1} \vee \tau_{2}$ is locally compact if and only if $\tau_{1} \wedge \tau_{2}$ is Hausdorff.
\end{abstract}

If $G$ is a group, the collection of all topologies that make $G$ into a topological group forms a lattice when set inclusion is used as a partial ordering. As is pointed out in [4], the intersection of two group topologies need not be a group topology. Thus this lattice of group topologies is not a sublattice of the lattice of all topologies on $G$. Recently some results concerning the structure of this lattice have been obtained in $[\mathbf{1}, \mathbf{2}$, and 3]. The purpose of this paper is to further study this lattice and to obtain relationships between the meet and join operators when $G$ is an abelian group. Hence we shall assume as additional hypothesis throughout the paper that $G$ is an abelian group.

THEOREM 1. If $\tau_{1}$ and $\tau_{2}$ are Hausdorff group topologies and $\tau_{1} \vee \tau_{2}$ is the discrete topology, then $\tau_{1} \wedge \tau_{2}$ is a Hausdorff topology.

ProOF. Endow $G \times G$ with the product topology $\tau_{1} \times \tau_{2}$. The map $m: G \times G \rightarrow G$ defined by $m\left(g_{1}, g_{2}\right)=g_{1} g_{2}$ is a homomorphism and the resulting quotient topology on $G$ is $\tau_{1} \wedge \tau_{2}$. The antidiagonal $\Delta=m^{-1}(e)$ has $\tau_{1} \vee \tau_{2}$ for its inherited topology.

Let $V_{1} \times V_{2}$ be a basic open set in $G \times G$ such that $\left(V_{1} \times V_{2}\right) \cap \Delta \neq \varnothing$. Then we have that $H \subset V_{1} \cdot V_{2}$, where $H=\{\bar{e}\}$. Let $z \in H-\{e\}$. We can find $(x, y) \in V_{1} \times V_{2}$ with $z=x y$. Hence, $(x, y)$ is a limit point of $\Delta$ in $G \times G$ and since $G_{1} \times G_{2}$ is Hausdorff, $\left(V_{1} \times V_{2}\right) \cap \Delta$ is infinite. Therefore, $\tau_{1} \vee \tau_{2}$ cannot be the discrete topology.

COROLLARY 1. Let $\tau_{1}$ be a minimal Hausdorff group topology and $\tau_{2}$ a Hausdorff group topology. If $\tau_{1} \vee \tau_{2}$ is discrete, then $\tau_{2}$ is discrete.

Theorem 1 also provides a partial answer to a question asked by Willard [5] concerning the intersection topology obtained from two Hausdorff topologies.

COROLLARY 2. If $\tau_{1}$ and $\tau_{2}$ are Hausdorff group topologies and $\tau_{1} \vee \tau_{2}$ is discrete, then $\tau_{1} \cap \tau_{2}$ is a Hausdorff topology.

THEOREM 2. Let $P$ be a topological property preserved by finite products and inherited by closed sets. If $\tau_{1}$ and $\tau_{2}$ have property $P$ and $\tau_{1} \wedge \tau_{2}$ is Hausdorff, then $\tau_{1} \vee \tau_{2}$ has property $P$.

Received by the editors November 19, 1985.

1980 Mathematics Subject Classification (1985 Revision). Primary 22A05, 54H99. 
ProOF. As in Theorem 1 we have $m:\left(G \times G, \tau_{1} \times \tau_{2}\right) \rightarrow\left(G, \tau_{1} \wedge \tau_{2}\right)$ a quotient map. Since $\{e\}$ is closed in $\left(G, \tau_{1} \wedge \tau_{2}\right)$, we have that the kernel of $m, \Delta$, is closed in $\left(G \times G, \tau_{1} \times \tau_{2}\right)$.

COROLLARY 3. If $\tau_{1}$ and $\tau_{2}$ are locally compact Hausdorff group topologies, then $\tau_{1} \vee \tau_{2}$ is locally compact if and only if $\tau_{1} \wedge \tau_{2}$ is Hausdorff.

\section{REFERENCES}

1. B. Clark and V. Schneider, The normal extensions of subgroup topologies, Proc. Amer. Math. Soc. 97 (1986), 163-166.

2. M. Lamper, Complements in the lattice of all topologies of topological groups, Arch. Math. (Brunensis) 4 (1974), 221-230.

3. D. Remus, Zur Struktur des Verbandes der Gruppentopologien, Dissertation, Universität Hannover, 1983.

4. P. Samuel, Ultrafilters and compactifications of uniform spaces, Trans. Amer. Math. Soc. 64 (1948), 100-134.

5. S. Willard, General topology, Addison-Wesley, Reading, Mass., 1970.

DEPARTMENT OF MATHEMATICS, UNIVERSity OF SOUTHWESTERN LOUisianA, LAFAYETTE, LOUISIANA 70504 